\title{
Outcome Analysis by Voice Assessment and Videostroboscopy in Laryngopharyngeal Reflux
}

\author{
Aparaajita Upadhyay ${ }^{1}$, Anshu Singh ${ }^{2}$, Anubhav Shrivastava ${ }^{3}$, Raj Kumar Mundra ${ }^{4}$
}

\begin{abstract}
Introduction: Change in voice in the patient of laryngopharyngeal reflux is a common symptom. In this study, we are assessing the improvement in severity of the laryngopharyngeal reflux disease by its symptoms, signs, severity, and videostroboscopy to detect even earliest change and document it.

Materials and methods: A prospective study in 50 subjects at a tertiary referral center who were subjected to answer a questionnaire of the voice handicap index, the reflux symptom index, and assessed reflux finding score and vocal fold mucosa changes by videostroboscopy. All subjects were reassessed after 12 weeks of treatment.

Result: Improvement was found statistically significant $(<0.05)$ in all parameters and scales.

Conclusion: Preventing the insult by acid reflux in the laryngeal mucosa by proton pump inhibitors resulted in improvement of symptoms and signs, which proves the efficacy of treatment. Videostroboscopy is a useful tool to early diagnose the lesion.

Keywords: Hoarseness, Laryngopharyngeal reflux, Reflux finding, Reflux symptom index, Voice assessment, Voice handicap index.

International Journal of Phonosurgery \& Laryngology (2019): 10.5005/jp-journals-10023-1176
\end{abstract}

\section{INTRODUCTION}

The retrograde movement of gastric contents into the larynx, the pharynx, and the upper aerodigestive tract is called laryngopharyngeal reflux (LPR). It is known to produce symptoms like hoarseness, globus, dysphagia, otalgia, and laryngospasm and may result in contact granulomata, esophageal webs, and pachyderma. ${ }^{1}$ Most of time this phenomenon can result in very subtle finding like arytenoid erythema, which is extremely commonly seen in the laryngology practice. ${ }^{1}$ This condition is prevalent in a large number of patients seeking laryngology consult for various indirect symptoms.

The objective of this study is to assess the etiological role of laryngopharyngeal reflux (LPR) in patients with change in voice and analyze the effectiveness of 3 months antireflux medication along with voice therapy.

\section{Materials and Methods}

This study was conducted at the ENT Department, MGM medical college, Indore. It is a prospective study designed to study objectively the etiological role of LPR in patient presenting with change in voice or hoarse voice and to analyze the effect of antireflux medication using the voice handicap index for the voice prognosis parameter, the reflux symptom index (RSI) for the LPR symptom indicator, and the reflux finding score (RFS) and stroboscopic parameter to assess the signs and improvement objectively.

A total of 50 patients were selected with the chief complaint of change in voice, and clinical diagnosis of laryngopharyngeal reflux was confirmed by the RSI (Table 1) and the RFS (Table 2), both initially developed by Belafsky et al. ${ }^{2}$ patients with RSI $>13$ and RFS $>7$ were enrolled. All patients were prospectively evaluated before treatment and 3 months after treatment with proton pump inhibitors twice daily, stream inhalation, and voice therapy.

Each patient underwent a comprehensive history taking, filling a simple questionnaire form for RSI and physical
'Department of ENT, Netaji Subhash Chandra Bose Medical College, Jabalpur, Madhya Pradesh, India

${ }^{2-4}$ Department of Otorhinolaryngology, Mahatma Gandhi Memorial Medical College, Indore, Madhya Pradesh, India

Corresponding Author: Anshu Singh, Department of Otorhinolaryngology, Mahatma Gandhi Memorial Medical College, Indore, Madhya Pradesh, India, Phone: +91 9561309646, e-mail: anshusingh2107@gmail.com

How to cite this article: Upadhyay A, Singh A, Shrivastava A, et al. Outcome Analysis by Voice Assessment and Videostroboscopy in Laryngopharyngeal Reflux. Int J Phonosurg Laryngol 2019;9(2): 25-29.

Source of support: Nil

Conflict of interest: None

examination including rigid laryngoscopy with stroboscopy and laryngeal photodocumentation and determination of the RFS at every visit.

Improvement in RSI, VHI, RFS, and stroboscopic parameters was assessed. The paired sample $t$ test and the Chi-square test were used for assessment of the above criteria, respectively.

\section{Results}

The mean age of group was 34.4 years with $40 \%$ patients were middle-aged between 25 years and 45 years.

Male preponderance with $56 \%$ patients were males.

The most common primary complaints were dysphonia $(n=50$, $100 \%)$, throat clearing $(n=38,76 \%)$, chest pain/heartburn/stomach disorder(s) ( $n=33,66 \%)$, and concerned cough ( $n=18,36 \%)$. The spectrum of laryngeal pathology is shown in Figure 1.

The mean RSI for the pretreatment group was $20.68 \pm$ 5.05 , which was significantly higher than the mean RSI for the posttreatment group $(9.32 \pm 4.16)$. 
Table 1: Reflux finding score

\begin{tabular}{ll}
\hline Subglottic edema & $0=$ Absent \\
& $2=$ Present \\
& $2=$ Partial \\
Ventricular & $4=$ Complete \\
& $2=$ Arytenoids only \\
Erythema/hyperemia & $4=$ Diffuse \\
Vocal fold edema & $1=$ Mild \\
& $2=$ Moderate \\
& $3=$ Severe \\
& $4=$ Polypoid \\
& $1=$ Mild \\
& $2=$ Moderate \\
Diffuse laryngeal edema & $3=$ Severe \\
& $4=$ Obstructing \\
Posterior commissure hypertrophy & $1=$ Mild \\
& $2=$ Moderate \\
& $3=$ Severe \\
$4=$ Obstructing \\
$0=$ Absent \\
$2=$ Present \\
$0=$ Absent \\
$2=$ Present \\
Granuloma/granulation tissue &
\end{tabular}

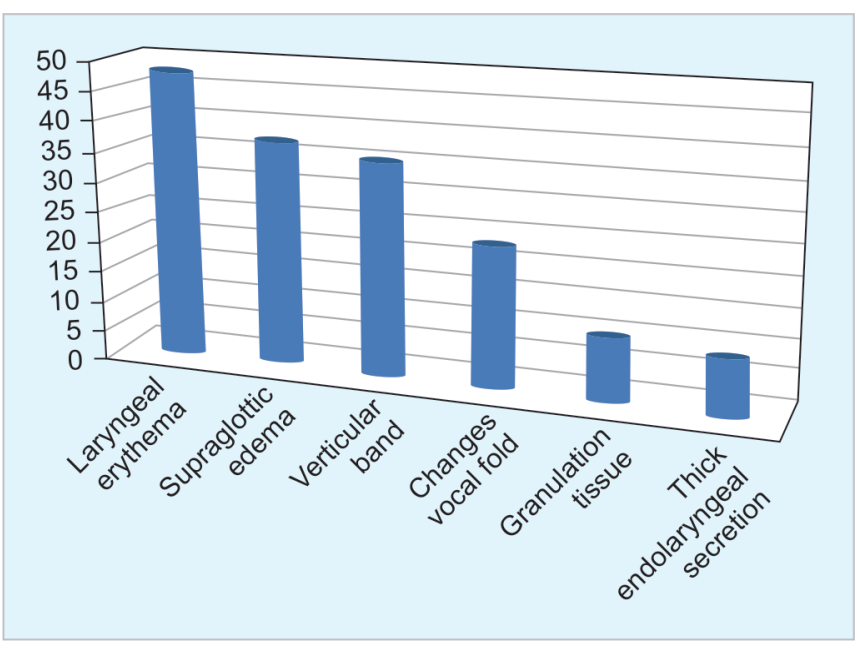

Fig. 1: Spectrum of laryngeal pathology in the study

The mean $\mathrm{VHI}$ for pretreatment group was $34 \pm 4.6$, which was significantly higher than the mean $\mathrm{VHI}$ for the posttreatment group (19.04 \pm 3.50$)$.

The mean value of RFS was $10.73 \pm 2.24$ in the pretreatment group and decreased significantly $(4.61 \pm 3.20)$ in the posttreatment group.

The videostroboscopic analysis showed an asymmetric mucosal wave in 23 patients out of which 12 patients had reactive lesions over the vocal fold, 7 had sulcus, 5 patients has nodule, and after treatment the asymmetry was found only in 4 patients. There was focal absence of the mucosal wave in 14 patients; this may be due to extension of insult due to acid reflux involving layers deeper than the vocal fold mucosa or due to bulky lesion restricting mobility of mucosa; after treatment the focal asymmetry of mucosal wave still
Table 2: Reflux symptom index

Within the last month, how did the following $0=$ No problem, problems affect you? $5=$ Severe problem

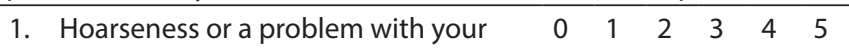
voice

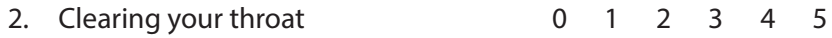

3. Excess throat mucus or postnasal drip $\begin{array}{lllllll}0 & 1 & 2 & 3 & 4 & 5\end{array}$

4. Difficulty swallowing food, liquids, or $\begin{array}{lllllll}0 & 1 & 2 & 3 & 4 & 5\end{array}$ pills

5. Coughing after eating or after lying $\quad \begin{array}{lllllll}0 & 1 & 2 & 3 & 4 & 5\end{array}$ down

6. Breathing difficulties or choking $\quad \begin{array}{lllllll}0 & 1 & 2 & 3 & 4 & 5\end{array}$ episodes

7. Troublesome or annoying cough $\quad \begin{array}{lllllll}0 & 1 & 2 & 3 & 4 & 5\end{array}$

8. Sensations of something sticking in $\begin{array}{lllllll}0 & 1 & 2 & 3 & 4 & 5\end{array}$ your throat or a lump in your throat

9. Heartburn, chest pain, indigestion, or $\begin{array}{lllllll}0 & 1 & 2 & 3 & 4 & 5\end{array}$ stomach acid coming up

found in 4 patients (Figs 2 to 8 ). Incomplete glottic closure was found in 38 patients mostly due to laryngeal edema result in incomplete touching of vocal folds, reactive lesion elevated margins touching early, nodules making hourglass shape closure, it reduced to only six patients after treatment, which was statistically significant improvement.

Therefore, the clinical assessments demonstrated an important improvement characterized by a significant decrease in $\mathrm{VHI}(p<$ $0.001), \operatorname{RSI}(p<0.001), \operatorname{RFS}(p<0.001)$, and stroboscopic parameters after 12 weeks of treatment (Table 3 ).

In 50 patients included in the study, 48 (96\%) patients had erythema/hyperemia involving the mucosa of the supraglottis mostly arytenoids and posterior commissure. Supraglottic edema was found in 37 (74\%) cases involving the mucosa over arytenoids and false vocal folds. Posterior ventricular bands were found in $70 \%$ of patients.

Around $46 \%$ patients had inflammatory changes over the vocal fold, which include vocal fold edema, leukoplakic patches, nodules, and sulcus. The granulation tissue was found in $20 \%$ of patients mostly involving posterior commissure, which is suggestive of chronic insult by the irritant, which is most likely to be acid reflux in this study. Around $18 \%$ of patients show thick endolaryngeal secretion.

\section{Discussion}

Laryngopharyngeal reflux is a common cause of voice impairment and chronic laryngitis. In past three decades, many studies have investigated the LPR signs and symptoms, diagnosis, medical and surgical treatments, and pathophysiological mechanisms underlying the development. But due to poor and inaccurate documentation and lack of observation by practitioners, voice disorders tend to be prevalent and also disabling for patients. Thus, Lechien et al. ${ }^{3}$ in his studies demonstrated significant differences in LPR patients concerning subjective (dysphonia and $\mathrm{VHI}$ ) and objective (aerodynamic and acoustic) voice assessments in comparison with healthy subjects. The study of $\mathrm{pH}$ monitoring done by Belafsky et al. ${ }^{4}$ developed RSI and RFS ${ }^{2}$ for the diagnosis and follow-up of LPR signs and symptoms. Park et al. ${ }^{5}$ found both scales are readily 
administered, highly reproducible, and exhibit excellent constructand criterion-based validity. In this study, we found that RSI and RFS improved significantly after 12 weeks of PPIs and steam inhalation and voice therapy. These findings are in accordance with previous studies by Jin et al., ${ }^{6}$ Masaany et al., ${ }^{7}$ Naiboglu et al., ${ }^{8}$ Chun et al., ${ }^{9}$ and Lechien et al., ${ }^{3}$ which observed the decrease in RSI and RFS after $\mathrm{PPI}$ and diet treatment.
In this study, we did not use $\mathrm{pH}$ meter for confirmation of reflux due to many limitations like inconvenient for large number of subject, cost and availability of equipment, and lack of consent for such test. We took history and chief complains as criteria for subjects and clinical scales proposed by Belafsky et al.

Voice change was one of the chief complaints we consider in this study. We took VHI as a parameter to assess voice improvement
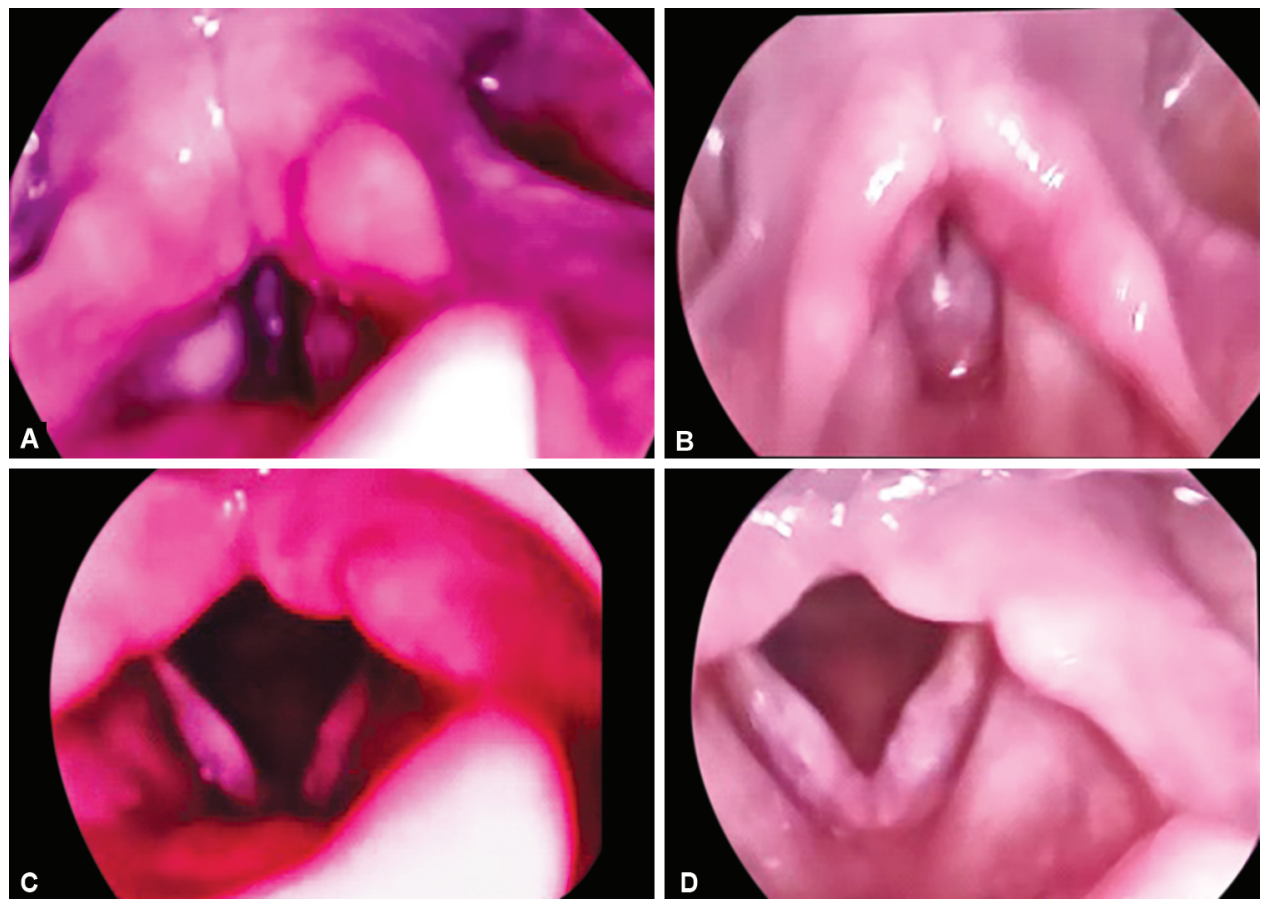

Figs. 2A to D: Generalized hyperemia, edema and granular changes improved after 3 months of treatment
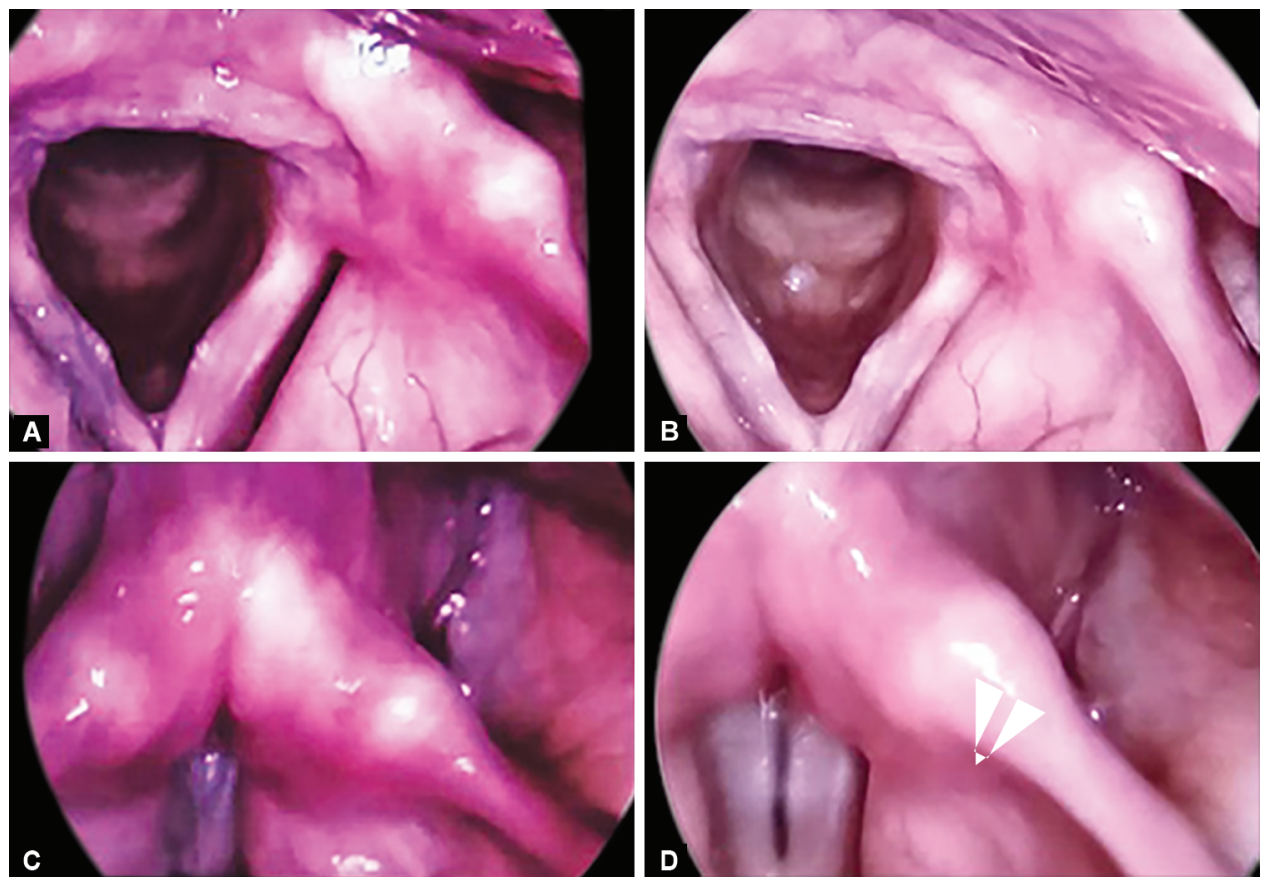

Figs. 3A to D: Reactionary lesion over vocal fold improved after treatment 


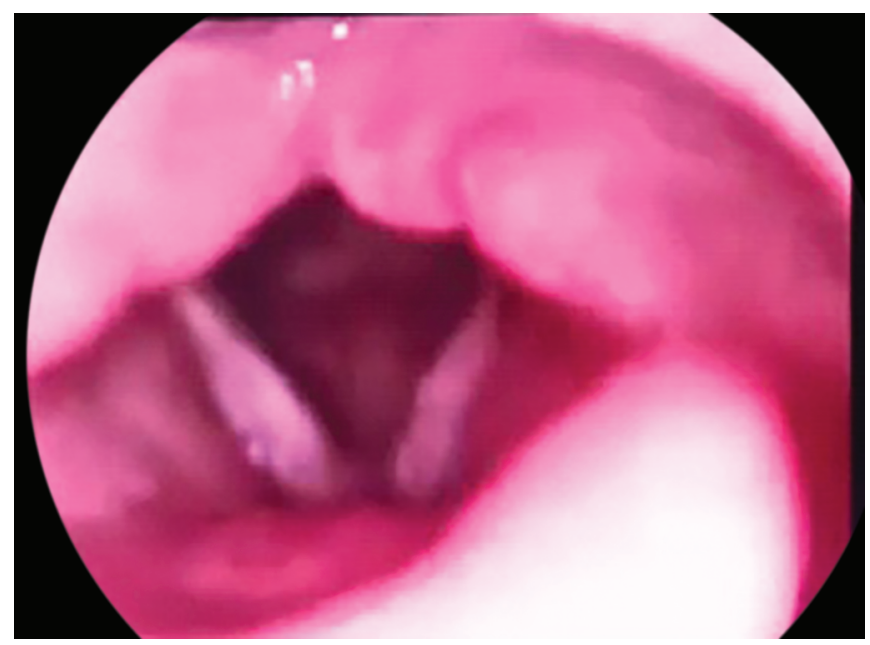

Fig. 4: Significant laryngeal edema and erythema

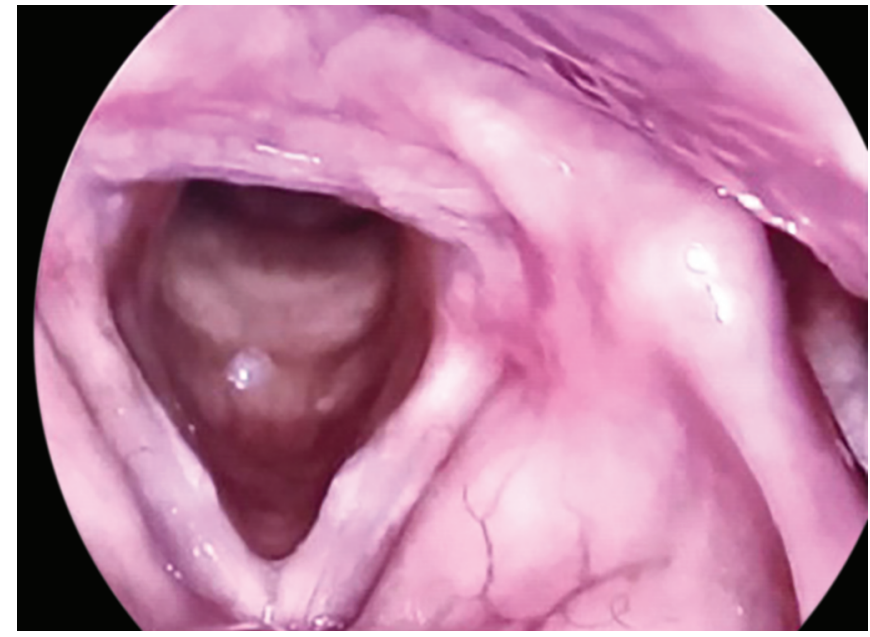

Fig. 6: Posterior web and reactive lesion over vocal fold

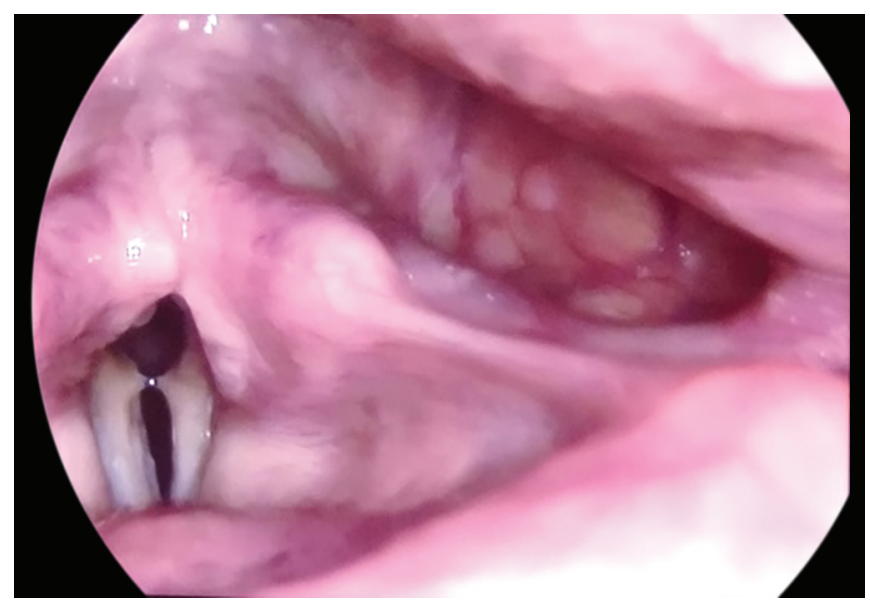

Fig. 8: Thick endolaryngeal secretion

after treatment and found almost 15 score difference in the pretreatment and the posttreatment group (pretreatment group was $34 \pm 4.6$, which was significantly higher than the mean RSI for the posttreatment group, $19.04 \pm 3.50$ ). Siupsinkiene et al. ${ }^{10}$ also reported the interest to use $\mathrm{VHI}$ as an outcome of the efficacy of the PPI treatment in LPR patients. Lechien et al. ${ }^{3}$ reported similar

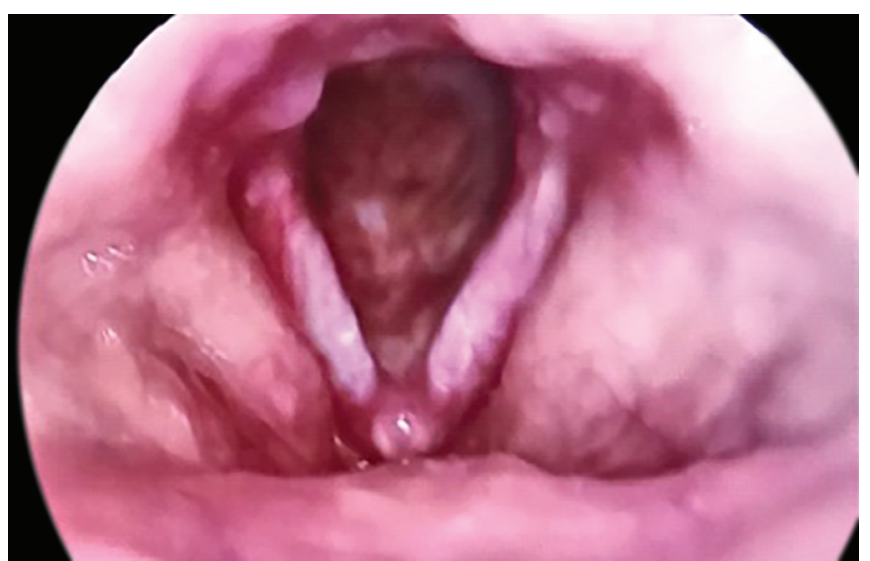

Fig. 5: Erythema over vocal folds

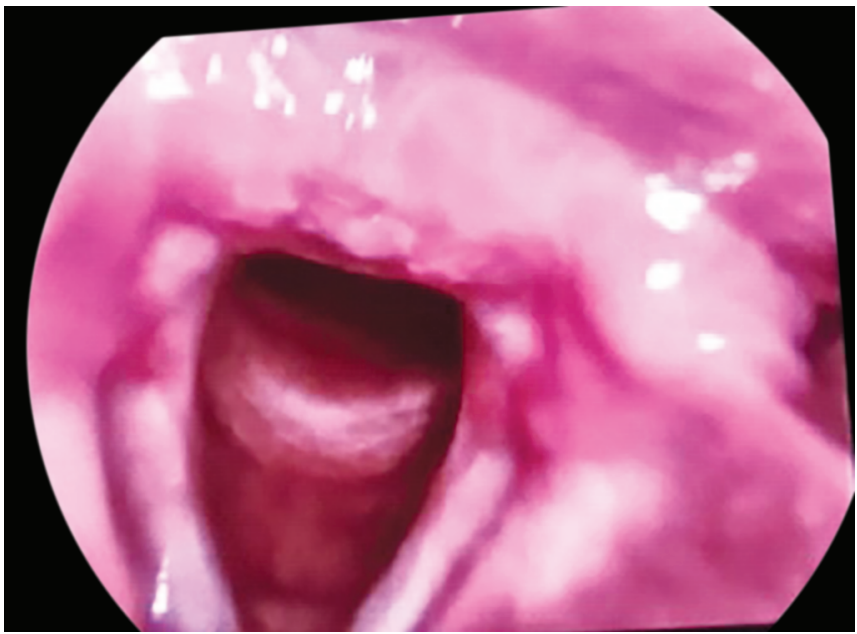

Fig. 7: Granulation tissue over interarytenoid region

Table 3: Summarized result of parameters before and after treatment

\begin{tabular}{|c|c|c|c|}
\hline Scales & Pretreatment & Posttreatment & $p$ value* \\
\hline RSI & $20.68 \pm 5.05$ & $9.32 \pm 4.16$ & $<0.001$ \\
\hline $\mathrm{VHI}$ & $34 \pm 4.6$ & $19.04 \pm 3.50$ & $<0.001$ \\
\hline RFS & $10.73 \pm 2.24$ & $4.61 \pm 3.20$ & $<0.001$ \\
\hline $\begin{array}{l}\text { Mucosal wave } \\
\text { asymmetry }\end{array}$ & 23 & 4 & $<0.001$ \\
\hline $\begin{array}{l}\text { Absence of mucosal } \\
\text { wave }\end{array}$ & 14 & 4 & $<0.001$ \\
\hline $\begin{array}{l}\text { Incomplete glottis } \\
\text { closure }\end{array}$ & 18 & 6 & $<0.001$ \\
\hline
\end{tabular}

*Improvement in patients after treatment

results in VHI in LPR patient after 12 weeks of therapy and diet modification.

Videostroboscopy was used to assess laryngoscopic finding and detect changes in the vocal fold due to LPR disease; RFS was done in the same sitting. Out of 50 subjects in the study, 38 had incomplete glottis closure and 23 of them had impaired mucosal movement.

Arytenoid erythema was the most common finding (97\%), followed by vocal cord erythema (94\%), arytenoid edema (93\%), posterior web $(76 \%)$, and posterior half vocal fold reactive lesion 
(24\%). Results are similar to Book et al. arytenoid erythema (97.5\%), vocal cord erythema (95.7\%) and edema (95.7\%), posterior commissure hypertrophy (94.9\%), and arytenoid edema (94.0\%). ${ }^{11}$

In this study, we found statistically significant improvement in RFS as well as videostroboscopy measures for LPR. Beaver et al. ${ }^{12}$ used the LPRD index, but he reported improvement in LPRD included supraglottic edema and erythema, glottic edema and erythema, and subglottic edema and erythema after 6 weeks of treatment, which is similar to our study.

\section{Conclusion}

Reflux may be present in up to $50 \%$ of patients with voice disorders. ${ }^{2}$ The ability of assessment of improvement after interval in patients with LPR relies on the clinician's ability to record laryngeal findings. ${ }^{13}$ The videostroboscopic assessment in patient with LPR gives a vivid picture of the disease spectrum and diagnoses the changes in the larynx at very early stage. In this digital era with quick availability of previous record, follow-up at frequent intervals gives idea of response to treatment. This study shows that 12-week therapy with PPI along with voice therapy and steam inhalation improves the signs of insults in larynx caused by LPR significantly.

\section{References}

1. Chodosh PL. Gastro-esophago-pharyngeal reflux. Laryngoscope 1977;87(9 Pt 1):1418-1427. DOI: 10.1288/00005537-19770900000002.

2. Belafsky PC, Postma GN, Koufman JA. The validity and reliability of the reflux. Laryngoscope 2001;111(8):1313-1317. DOI: 10.1097/00005537200108000-00001.

3. Lechien JR, Huet K, Khalife M, et al. Impact of laryngopharyngeal reflux on subjective and objective voice assessments: a prospective study. J Otolaryngol Head Neck Surg [Internet] 2016;45(1):59. DOI:
10.1186/s40463-016-0171-1Available from https://www.ncbi.nlm nih.gov/pmc/articles/PMC5101798/.

4. Belafsky PC, Postma GN, Koufman JA. Validity and reliability of the reflux symptom index (RSI). J Voice 2002;16(2):274-277. DOI: 10.1016/ s0892-1997(02)00097-8.

5. Park KH, Choi SM, Kwon SUK, et al. Diagnosis of laryngopharyngeal reflux among globus patients. Otolaryngol Head Neck Surg 2006;134(1):81-85. DOI: 10.1016/j.otohns.2005.08.025.

6. Jin BJ, Lee YS, Jeong SW, etal.Change of acoustic parameters before and after treatment in laryngopharyngeal reflux patients. Laryngoscope 2008;118(5):938-941. DOI: 10.1097/MLG.0b013e3181651c3a.

7. Masaany M, Marina MB, Sharifa Ezat WP, et al. Empirical treatment with pantoprazole as a diagnostic tool for symptomatic adult laryngopharyngeal reflux. J Laryngol Otol 2011;125(5):502-508. DOI: 10.1017/S0022215111000120.

8. Naiboglu B, Durmus R, Tek A, et al. Do the laryngopharyngeal symptoms and signs ameliorate by empiric treatment in patients with suspected laryngopharyngeal reflux? Auris Nasus Larynx 2011;38(5):622-627. DOI: 10.1016/j.anl.2011.01.010.

9. Chun B-J, Lee D-S. The effect of itopride combined with lansoprazole in patients with laryngopharyngeal reflux disease. Eur Arch Otorhinolaryngol 2013;270(4):1385-1390. DOI: 10.1007/s00405-0122341-8.

10. Siupsinskiene N, Adamonis K, Toohill RJ. Quality of life in laryngopharyngeal reflux patients. Laryngoscope 2007;117(3): 480-484. DOI: 10.1097/MLG.0b013e31802d83cf.

11. Book DT, Rhee JS, Toohill RJ, et al. Perspectives in laryngopharyngeal reflux: an international survey. Laryngoscope 2002;112(8):1399-1406. DOI: 10.1097/00005537-200208000-00014.

12. Beaver ME, Stasney CR, Weitzel E, et al. Diagnosis of laryngopharyngeal reflux disease with digital imaging. Otolaryngol Head Neck Surg 2003;128(1):103-108. DOI: 10.1067/mhn.2003.10.

13. Raghunandhan S, Nagasundaram J, Natarajan K, et al. Videostroboscopy in laryngopharyngeal reflux disorder. Murthy $\mathrm{P}_{\text {, }}$ ed. Int J Phonosurg Laryngol 2011;1(2):52-56. DOI: 10.5005/ jp-journals-10023-1013. 